\title{
An Artificial Bee Colony Algorithm for the Capacitated Vehicle Routing Problem
}

\author{
W. Y. Szeto ${ }^{1,2}$, Yongzhong $\mathrm{Wu}^{3}$ and $\mathrm{Sin} \mathrm{C} . \mathrm{Ho}^{4}$ \\ ${ }^{2}$ Department of Civil Engineering, The University of Hong Kong, Pokfulam Road, \\ Hong Kong, PR China \\ ${ }^{3}$ Department of Industrial Engineering, South China University of Technology, PR China \\ ${ }^{4}$ CORAL, Department of Business Studies, School of Business and Social Sciences, \\ Aarhus University, Denmark
}

\begin{abstract}
This paper introduces an artificial bee colony heuristic for solving the capacitated vehicle routing problem. The artificial bee colony heuristic is a swarm-based heuristic, which mimics the foraging behavior of a honey bee swarm. An enhanced version of the artificial bee colony heuristic is also proposed to improve the solution quality of the original version. The performance of the enhanced heuristic is evaluated on two sets of standard benchmark instances, and compared with the original artificial bee colony heuristic. The computational results show that the enhanced heuristic outperforms the original one, and can produce good solutions when compared with the existing heuristics. These results seem to indicate that the enhanced heuristic is an alternative to solve the capacitated vehicle routing problem.
\end{abstract}

Keywords: Routing, Artificial Bee Colony, Metaheuristic

\footnotetext{
${ }^{1}$ Corresponding author. Tel.: +852 2857 8552; fax: +852 2559 5337. Email address: ceszeto@hku.hk
} 


\section{Introduction}

The Capacitated Vehicle Routing Problem (CVRP) (i.e., the classical vehicle routing problem) is defined on a complete undirected graph $G=(V, E)$, where $V=\{0,1, \ldots, n\}$ is the vertex set and $E=\{(i, j): i, j \in V, i<j\}$ is the edge set. Vertices $1, \ldots, n$ represent customers; each customer $i$ is associated with a nonnegative demand $d_{i}$ and a nonnegative service time $s_{i}$. Vertex 0 represents the depot at which a fleet of $m$ homogeneous vehicles of capacity $Q$ is based. The fleet size is treated as a decision variable. Each edge $(i, j)$ is associated a nonnegative traveling cost or travel time $c_{i j}$. The CVRP is to determine $m$ vehicle routes such that (a) every route starts and ends at the depot; (b) every customer is visited exactly once; (c) the total demand of any vehicle route does not exceed $Q$; and (d) the total cost of all vehicle routes is minimized. In some cases, the CVRP also imposes duration constraints where the duration of any vehicle route must not exceed a given bound L. Mathematical formulations of the CVRP can be found in Toth and Vigo (2002).

As the CVRP is a NP-hard problem, only instances of small sizes can be solved to optimality using exact solution methods (e.g., Toth and Vigo, 2002; Baldacci et al., 2010), and this might not even be possible if it is required to use limited amount of computing time. As a result of this, heuristic methods are used to find good, but not necessarily guaranteed optimal solutions using reasonable amount of computing time. During the past two decades, an increasing number of publications on heuristic approaches have been developed to tackle the CVRP. The work can be categorized into evolutionary algorithms (Baker and Ayechew, 2003; Berger and Barkaoui, 2003; Prins, 2004; Mester and Bräysy, 2007; Prins, 2009; Nagata and Bräysy, 2009), ant colony optimization (Bullnheimer et al., 1999; Reimann et al., 2004; Yu et al., 2009), simulated annealing (Osman, 1993; Lin et al., 2009), tabu search (Taillard, 1993; Gendreau et al., 1994; Rego and Roucairol, 1996; Rego, 1998; Cordeau et al., 2001; Toth and Vigo, 2003; Derigs and Kaiser, 2007), path-relinking (Ho and Gendreau, 2006), adaptive memory procedures (Rochat and Taillard, 1995; Tarantilis and Kiranoudis, 2002; Tarantilis, 2005), large neighborhood search (Ergun et al., 2006; Pisinger and Ropke, 2007), variable neighborhood search (Kytöjoki et al., 2007; Chen et al., 2010), deterministic annealing (Golden et al., 1998; Li et al., 2005), honey-bees mating optimization (Marinakis et al., 2010), particle swarm optimization (Ai and Kachitvichyanukul, 2009) and hybrid Clarke and Wright's savings heuristic (Juan et al., 2010).

Some of the above CVRP heuristics are based on previously developed heuristics. In the following we will mention some of the relationships. Rochat and Taillard's (1995) adaptive memory 
procedure used the idea of genetic algorithms of combining solutions to construct new solutions and employed the tabu search of Taillard (1993) as an improvement procedure. Tarantilis (2005) had a similar framework as Tarantilis and Kiranoudis (2002) (which is a variant of the idea proposed by Rochat and Taillard), but is different in that it does not rely on probability as in Tarantilis and Kiranoudis (2002) and also uses a more sophisticated improvement procedure. The Unified tabu search (Cordeau et al., 2001) and the tabu search used by Ho and Gendreau (2006) contain some of the features found in Taburoute (Gendreau et al., 1994); infeasible solutions are considered by extending the objective function with a penalty function and the use of continuous diversification. Granular tabu search (Toth and Vigo, 2003) restricts the neighborhood size by removing edges from the graph that are unlikely to appear in an optimal solution. Later, other researchers have also applied this idea on their CVRP heuristics (e.g., Li et al., 2005; Mester and Bräysy, 2007; Chen et al., 2010). For extensive surveys on VRP metaheuristics, the reader is referred to Gendreau et al. (2002), Cordeau et al. (2005) and Gendreau et al. (2008).

While the above heuristics have been widely used and successfully applied to solve the CVRP for several years, Artificial Bee Colony $(\mathrm{ABC})$ is a fairly new approach introduced just a few years ago by Karaboga (2005), but has not yet been applied to solve the CVRP. However, ABC has been applied to solve other problems with great success (Baykasoğly et al., 2007; Kang et al., 2009; Karaboga, 2009; Karaboga and Basturk, 2007, 2008; Karaboga and Ozturk, 2009; Singh, 2009). It is worthwhile to evaluate the performance of the $\mathrm{ABC}$ algorithm for solving the CVRP.

In this paper, we evaluate the performance of the $\mathrm{ABC}$ heuristic using classical benchmark instances. An enhanced version of $\mathrm{ABC}$ heuristic is also proposed to improve the performance of the basic $\mathrm{ABC}$ heuristic for solving the CVRP. Computational results show that the enhanced $\mathrm{ABC}$ heuristic can produce much better solutions than the basic one, and the computation time of the enhanced $\mathrm{ABC}$ has slightly increased. The results obtained by the enhanced $\mathrm{ABC}$ heuristic are also competitive to those obtained from other metaheuristics. The paper is organized as follows. In Section 2, we give a review of the basic ABC algorithm. Refinements of the basic ABC algorithm and how they are applied to the CVRP are presented in Section 3. Experimental results showing the improvements in the performance of the enhanced ABC algorithm are presented in Section 4. Finally, Section 5 concludes the paper. 


\section{Artificial bee colony algorithm}

The ABC algorithm belongs to a class of evolutionary algorithms that are inspired by the intelligent behavior of the honey bees in finding nectar sources around their hives. This class of metaheuristics has only started to receive attention recently. Different variations of bee algorithms under various names have been proposed to solve combinatorial problems. But in all of them, some common search strategies are applied; that is, complete or partial solutions are considered as food sources and the groups of bees try to exploit the food sources in the hope of finding good quality nectar (i.e., high quality solutions) for the hive. In addition, bees communicate between themselves about the search space and the food sources by performing a waggle dance.

In the $\mathrm{ABC}$ algorithm, the bees are divided into three types; employed bees, onlookers and scouts. Employed bees are responsible for exploiting available food sources and gathering required information. They also share the information with the onlookers, and the onlookers select existing food sources to be further explored. When the food source is abandoned by its employed bee, the employed bee becomes a scout and starts to search for a new food source in the vicinity of the hive. The abandonment happens when the quality of the food source is not improved after performing a maximum allowable number of iterations.

The ABC algorithm is an iterative algorithm, and it starts by generating random solutions as the food sources and assigning each employed bee to a food source. Then during each iteration, each employed bee finds a new food source near its originally assigned (or old) food source (using a neighborhood operator). The nectar amount (fitness) of the new food source is then evaluated. If the new food source has more nectar than the old one, then the old one is replaced by the new one. After all employed bees have finished with the above exploitation process, they share the nectar information of the food sources with the onlookers. Then, each onlooker selects a food source according to the traditional roulette wheel selection method. After that, each onlooker finds a food source near its selected food source (using a neighborhood operator) and calculates the nectar amount of the neighbor food source. Then, for each old food source, the best food source among all the food sources near the old food source is determined. The employed bee associated with the old food source is assigned to the best food source and abandons the old one if the best food source is better than the old food source. A food source is also abandoned by an employed bee if the quality of the food source has not been improved for limit (a predetermined number) successive iterations. That employed bee then becomes a scout, and searches for new food source randomly. After the scout finds a new food source, the scout becomes an employed bee again. After each employed bee 
is assigned to a food source, another iteration of the $\mathrm{ABC}$ algorithm begins. The whole process is repeated until a stopping condition is met.

The steps of the $\mathrm{ABC}$ algorithm are summarized as follows:

1. Randomly generate a set of solutions as initial food sources $x_{i}, i=1, \ldots, \tau$. Assign each employed bee to a food source.

2. Evaluate the fitness $f\left(x_{i}\right)$ of each of the food sources $x_{i}, i=1, \ldots, \tau$.

3. Set $v=0$ and $l_{1}=l_{2}=\ldots=l_{\tau}=0$.

4. Repeat

a. For each food source $x_{i}$

i. Apply a neighborhood operator on $x_{i} \rightarrow \tilde{x}$.

ii. If $f(\tilde{x})>f\left(x_{i}\right)$, then replace $x_{i}$ with $\tilde{x}$ and $l_{i}=0$, else $l_{i}=l_{i}+1$.

b. Set $G_{i}=\varnothing, i=1, \ldots, \tau$, where $G_{i}$ is the set of neighbor solutions of food source $i$.

c. For each onlooker

i. Select a food source $x_{i}$ using the fitness-based roulette wheel selection method.

ii. Apply a neighborhood operator on $x_{i} \rightarrow \tilde{x}$.

iii. $G_{i}=G_{i} \cup\{\tilde{x}\}$

d. For each food source $x_{i}$ and $G_{i} \neq \varnothing$

i. Set $\hat{x} \in \arg \max _{\sigma \in G_{i}} f(\sigma)$.

ii. If $f\left(x_{i}\right)<f(\hat{x})$, then replace $x_{i}$ with $\hat{x}$ and $l_{i}=0$, else $l_{i}=l_{i}+1$.

e. For each food source $x_{i}$

i. If $l_{i}=$ limit , then replace $x_{i}$ with a randomly generated solution.

f. $v=v+1$

5. Until $(v=$ MaxIterations $)$

\section{Application to the CVRP}

In this section, different components of the algorithm are first explained, and then how they are woven together and applied to the CVRP is described. 


\subsection{Solution representation}

In order to maintain the simplicity of the $\mathrm{ABC}$ algorithm, a rather straightforward solution representation scheme is adopted. Suppose $n$ customers are visited by $m$ vehicle routes. The representation has the form of a vector of length $(n+m)$. In the vector, there are $n$ integers between 1 and $n$ inclusively representing customers' identity. There are also $m 0$ s in the vector representing the start of each vehicle route from the depot. The sequence between two 0s is the sequence of customers to be visited by a vehicle. Figure 1 illustrates a representation of a CVRP instance with $n=7$ and $m=3$. As shown in Figure 1, customers 4 and 1 are assigned to the same vehicle route and the vehicle visits customer 4 before customer 1.

\begin{tabular}{|l|l|l|l|l|l|l|l|l|l|}
\hline 0 & 4 & 1 & 0 & 2 & 7 & 5 & 0 & 3 & 6 \\
\hline
\end{tabular}

Figure 1. Solution representation

\subsection{Search space and cost functions}

The search may become restrictive if only the feasible part of the solution space is explored. Hence, we also allow the search to be conducted in the infeasible part of the solution space, so that the search can oscillate between feasible and infeasible parts of the solution space $X$. Each solution $x \in X$ consists of $m$ vehicle routes where each one of them starts and ends at the depot, and every customer is visited exactly once. Thus, $x$ may be infeasible with regard to the capacity and/or duration constraints. For a solution $x$, let $c(x)$ denote its travel cost, and let $q(x)$ and $t(x)$ denote the total violation of the capacity and duration constraints respectively. The routing cost of a vehicle $k$ corresponds to the sum of the costs $c_{i j}$ associated with the edges $(i, j)$ traversed by this vehicle. The total violation of the capacity and duration constraints is computed on a route basis with respect to $Q$ and $L$. Each solution $x$ found in the search is evaluated by a cost function $z(x)=c(x)+\alpha q(x)+\beta t(x)$ that includes the considerations mentioned above. The coefficients $\alpha$ and $\beta$ are self-adjusting positive parameters that are modified at every iteration. The parameter $\alpha$ is adjusted as follows: If the number of solutions with no violation of the capacity constraints is greater than $\tau / 2$, the value of $\alpha$ is divided by $1+\delta$, otherwise it is multiplied by $1+\delta$. The same rule also applies to $\beta$ with respect to the duration constraints. 


\subsection{Initial solution}

An initial solution is constructed by assigning one customer at a time to one of the $m$ vehicle routes. The selection of the customer is randomly made. The customer is then assigned to the location that minimizes the cost of assigning this customer over the current set of vehicle routes. The above procedure is repeated until all customers are routed.

A total of $\tau$ initial solutions are generated by the above procedure.

\subsection{Neighborhood operators}

A neighborhood operator is used to obtain a new solution $\tilde{x}$ from the current solution $x$ in Step 4 of the $\mathrm{ABC}$ heuristic. A number of neighborhood operators (among those listed below) are chosen in advance. Any combinations of the listed operators are possible, even one. Then, whenever a new solution $\tilde{x}$ is needed, a neighborhood operator is randomly chosen from the set of pre-selected neighborhood operators and applied once (i.e., no local search is performed) to the solution $x$. The set of pre-selected operators is determined by experimental testing discussed in Section 4.1. The possible operators include:

1. Random swaps

This operator randomly selects positions (in the solution vector) $i$ and $j$ with $i \neq j$ and swaps the customers located in positions $i$ and $j$. See Figure 2, where $i=3$ and $j=7$.

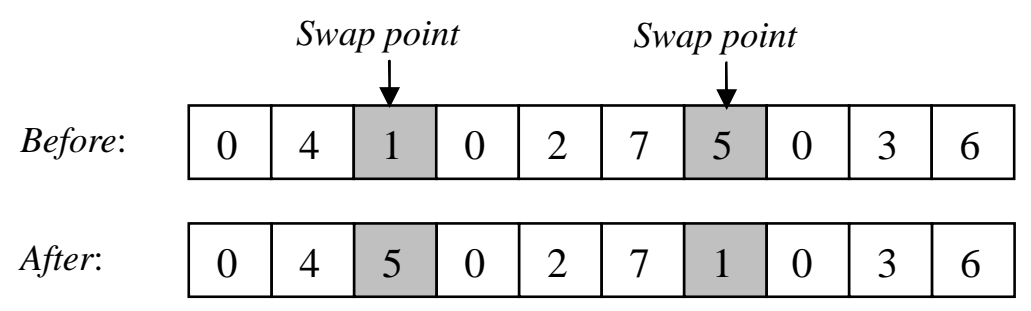

Figure 2. Random swaps

2. Random swaps of subsequences

This operator is an extension of the previous one, where two subsequences of customers and depot of random lengths are selected and swapped. An example is shown in Figure 3. 


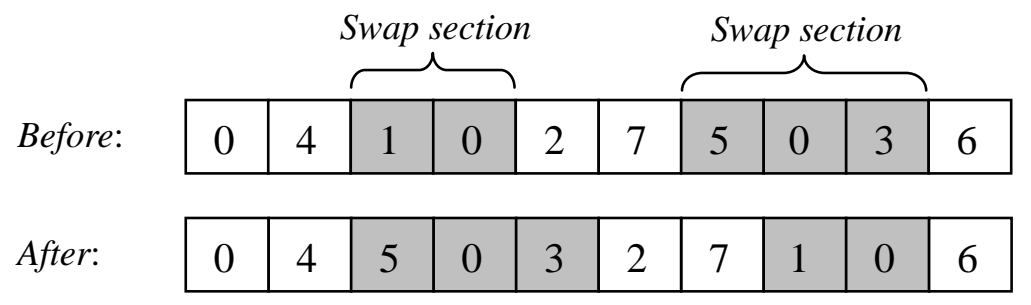

Figure 3. Random swaps of subsequences

3. Random insertions

This operator consists of randomly selecting positions $i$ and $j$ with $i \neq j$ and relocating the customer from position $i$ to position $j$. See Figure 4, where customer 5 is relocated from position 7 to position 3 .

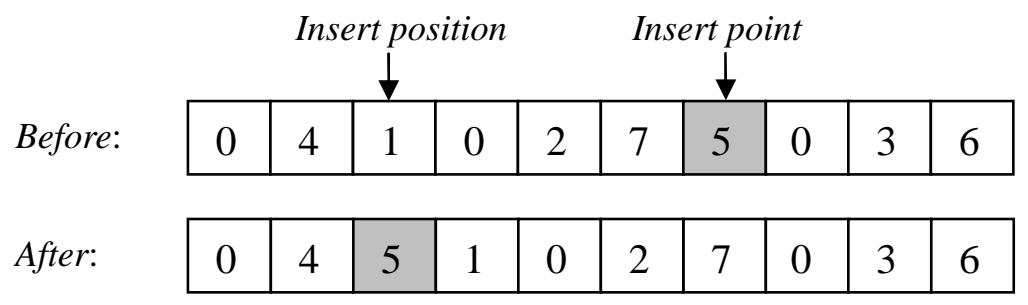

Figure 4. Random insertions

4. Random insertions of subsequences

This operator is an extension of the operator of random insertions where a subsequence of customers and depot of random length starting from position $i$ is relocated to position $j$. Positions $i$ and $j$ are randomly selected. Figure 5 gives an example of this operator.

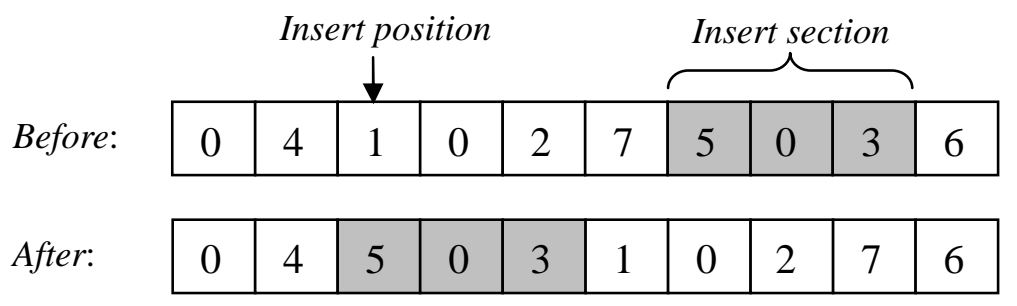

Figure 5. Random insertions of subsequences

5. Reversing a subsequence

A subsequence of consecutive customers and depot of random length is selected and then the order of the corresponding customers and depot is reversed, as shown in Figure 6. 


Before: \begin{tabular}{|l|l|l|l|l|l|l|l|l|l|}
0 & 4 & 1 & 0 & 2 & 7 & 5 & 0 & 3 & 6 \\
After: & \multicolumn{10}{|c|}{0} & 4 & 1 & 0 & 2 & 7 & 3 & 0 & 5 & 6 \\
\cline { 2 - 10 }
\end{tabular}

Figure 6. Reversing a subsequence

6. Random swaps of reversed subsequences

This operator is a combination of two previously mentioned operators. Two subsequences of customers and depot of random lengths are chosen and swapped. Then each of the swapped subsequences may be reversed with a probability of 50\%. An example of this operator is displayed in Figure 7.

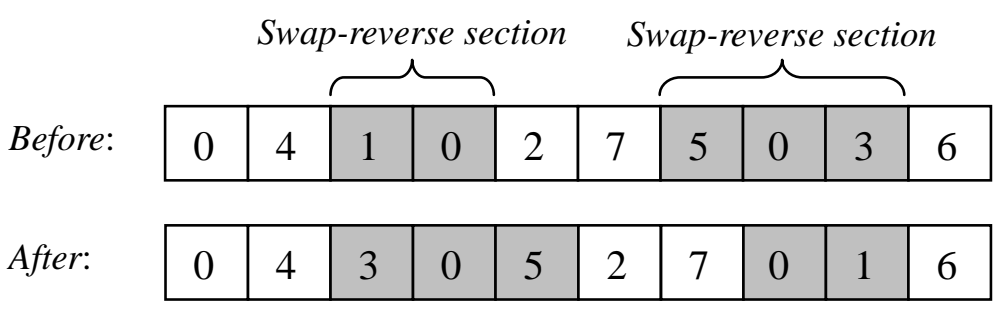

Figure 7. Random swaps of reversed subsequences

7. Random insertions of reversed subsequences

A subsequence of customers and depot of random length starting from position $i$ is relocated to position $j$. Then the relocated subsequence has a $50 \%$ chance of being reversed. Figure 8 shows an example of this operator where $i=7$ and $j=3$, and the subsequence is reversed.

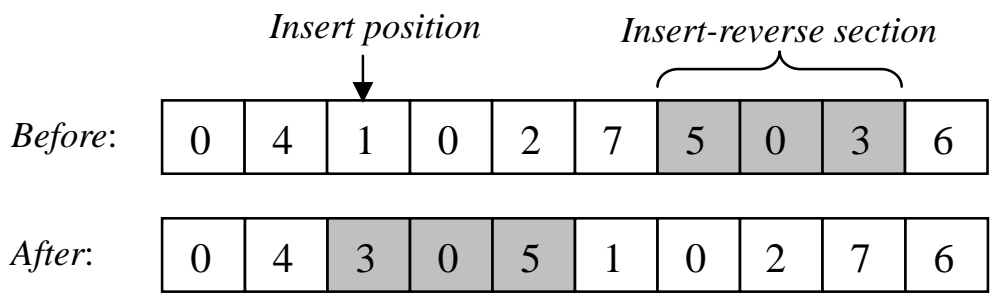

Figure 8. Random insertions of reversed subsequences 


\subsection{Selection of food sources}

At each iteration of the algorithm, each onlooker selects a food source randomly. In order to drive the selection process towards better food sources, we have implemented a roulette-wheel selection method for randomly selecting a food source. The probability of choosing the food source $x_{i}$ is then defined as $p\left(x_{i}\right)=\frac{f\left(x_{i}\right)}{\sum_{i=1}^{\tau} f\left(x_{i}\right)}, i=1, \ldots, \tau$ where $f\left(x_{i}\right)=\frac{1}{z\left(x_{i}\right)}$.

\subsection{An enhanced artificial bee colony algorithm}

It is shown in the literature that the basic $\mathrm{ABC}$ algorithm is capable of solving certain problems with great success. However, according to our computational experiments (see Section 4) this does not apply to the CVRP. Therefore, in this section, we will propose an enhanced version of the ABC algorithm to improve the performance of the basic ABC algorithm for solving the CVRP.

Step 4d of the basic ABC algorithm states that a food source $x_{i}$ will only be replaced by a neighbor solution $\hat{x}$ if $\hat{x}$ is of a better quality than $x_{i}$, where $\hat{x}$ is the best neighbor solution found by the onlookers in Step 4b. In the refined version, this condition is altered so that if the best neighbor solution $\hat{x}$ found by all the onlookers associated with food source $i$ is better than the food source $x_{i}$, then $\hat{x}$ will replace the food source $x_{j}$ that possesses the following two properties: 1) $x_{j}$ has not been improved for the largest number of iterations among all the existing food sources and 2) $x_{j}$ is worse than $\hat{x}$. In this way, potential food sources (i.e., the food sources which can produce better neighbor solutions) will be given opportunities to be further explored whereas non-potential food sources (i.e., the food sources which have not been improved for a relatively large number of iterations and are worse than new neighbor solutions) are excluded.

Step 4e of the basic ABC algorithm states that if no improving neighbor solutions of the food source $x_{i}$ have been identified during the last limit successive iterations, then $x_{i}$ is replaced by a randomly generated solution (using the procedure described in Section 3.3). In the refined version, this is modified so that $x_{i}$ is replaced by a new neighbor solution $\hat{x}$ of $x_{i}$. The quality of $\hat{x}$ can be worse or better than $x_{i}$. This modification may be beneficial as it prevents the search from searching in bad regions of the solution space with no control of the quality of the food sources.

The steps of the refined ABC algorithm are summarized below: 
1. Randomly generate a set of solutions as initial food sources $x_{i}, i=1, \ldots, \tau$.

Set $F=\cup_{i=1}^{\tau}\left\{x_{i}\right\}$, where $F$ is the set of food sources. Assign each employed bee to a food source.

2. Evaluate the fitness $f\left(x_{i}\right)$ of each of the food sources $x_{i}, i=1, \ldots, \tau$.

3. Set $v=0$ and $l_{1}=l_{2}=\ldots=l_{\tau}=0$.

4. Repeat

a. For each food source $x_{i}$

i. Apply a neighborhood operator on $x_{i} \rightarrow \tilde{x}$.

ii. If $f(\tilde{x})>f\left(x_{i}\right)$, then replace $x_{i}$ with $\tilde{x}$ and $l_{i}=0$, else $l_{i}=l_{i}+1$.

b. Set $G_{i}=\varnothing, i=1, \ldots, \tau$.

c. For each onlooker

i. Select a food source $x_{i}$ using the fitness-based roulette wheel selection method.

ii. Apply a neighborhood operator on $x_{i} \rightarrow \tilde{x}$.

d. $G_{i}=G_{i} \cup\{\tilde{x}\}$

e. For each food source $x_{i}$ and $G_{i} \neq \varnothing$

i. Set $\hat{x} \in \arg \max _{\sigma \in G_{i}} f(\sigma)$.

ii. If $f\left(x_{i}\right)<f(\hat{x})$, then select $\tilde{x}_{j} \in F$ with

$j \in \arg \max _{i=1, \ldots,|F|}\left\{l_{i} \mid f(\hat{x})>f\left(x_{i}\right)\right.$ and $\left.x_{i} \in F\right\}$, replace $\tilde{x}_{j}$ with $\hat{x}$, and $l_{i}=0$, else $l_{i}=l_{i}+1$.

f. For each food source $x_{i}$

i. If $l_{i}=$ limit , then apply a neighborhood operator on $x_{i} \rightarrow \tilde{x}$ and replace $x_{i}$ with $\tilde{x}$.

g. $v=v+1$

5. Until $(v=$ MaxIterations $)$

\section{Computational experiments}

We have tested both the original and the refined ABC heuristics on two standard benchmark 
instance sets. These include the fourteen classical Euclidean VRP and distance-constrained VRP instances described in Christofides and Eilon (1969) and Christofides et al. (1979) and the twenty large scale instances described in Golden et al. (1998). All experiments were performed on a 1.73 $\mathrm{GHz}$ computer, and the heuristics were coded in Visual C++ 2003.

The number of employed bees and the number of onlookers are set to be equal to the number of food sources $(\tau)$, which is 25 . This is based on Karaboga and Basturk (2008), which set the number of employed bees to be equal to the number of onlookers to reduce the number of parameters, and find that the colony size (i.e., the total number of employed bees and onlookers) of 50 can provide an acceptable convergence speed for search.

To reduce the number of parameters tuned, we let $\alpha=\beta$. We then evaluated the performance of the heuristics using different values of $\alpha$ in the interval [0.0001, 1] and $\delta=0.001$. It is found that the performance of the heuristics is insensitive over this range for many test instances, and for a majority of the test instances setting $\alpha=\beta$ to 0.1 seems to be the best choice. Based on these values of $\alpha$ and $\beta$, we tested the best value of $\delta$ over the range $[0.0001,1]$. The results show that the performance of the heuristics varies significantly in this interval for many test instances, and good solutions are obtained with $\delta=0.001$. Therefore, we set $\alpha=\beta=0.1$ and $\delta=0.001$ for all further experiments.

\subsection{Comparison of neighborhood operators}

In order to evaluate the effectiveness of each neighborhood operator, we have first tested the original $\mathrm{ABC}$ heuristic on instance vrpnc1 by considering each neighborhood operator at a time, including: 1. random swaps (swap); 2. random swaps of subsequences (section_swap); 3. random insertions (insert); 4. random insertions of subsequences (section_insert); 5. reversing a subsequence (reverse); 6. random swaps of reversed subsequences (swap_reverse); and 7. random insertions of reversed subsequences (insert_reverse). The results are reported in Table 1 . The best known solution reported in the literature for this instance is 524.61. Each of the seven variants was run 20 times, and each time the algorithm was run for 100,000 iterations. From the table, one can observe that the different neighborhood operators contribute differently. It can be seen that the ABC heuristic with the operator of randomly swapping reversed subsequences achieves the best average objective value, while random insertions and random insertions of subsequences did not yield promising results. Even though the variant of incorporating the operator of randomly swapping 
reversed subsequences did not lead to the best known solution, the average deviation from the best known solution is less than $1.5 \%$. Reversing a subsequence is another operator which also yields good performance, and uses less computation time than the operator of randomly swapping reversed subsequences.

Table 1. Experimental results by different operators for instance vrpnc1

\begin{tabular}{lllllllll}
\hline Operator & swap & $\begin{array}{l}\text { section_ } \\
\text { swap }\end{array}$ & insert & $\begin{array}{l}\text { section_ } \\
\text { insert }\end{array}$ & reverse & $\begin{array}{l}\text { swap_ } \\
\text { reverse }\end{array}$ & $\begin{array}{l}\text { insert_- } \\
\text { reverse }\end{array}$ & combined \\
\hline min. $^{\text {a }}$ & 576.47 & 550.94 & 758.12 & 623.79 & 528.56 & 527.82 & 534.41 & 526.97 \\
max $^{\text {b }}$ & 627.52 & 597.14 & 810.71 & 655.81 & 543.83 & 536.64 & 556.91 & 535.43 \\
avg $^{\text {c }}$ & 610.81 & 567.34 & 784.09 & 640.69 & 535.57 & 532.44 & 542.58 & 531.74 \\
std.dev. $^{\text {d }}$ & 15.98 & 10.89 & 14.01 & 8.60 & 4.62 & 4.43 & 6.01 & 3.58 \\
gap $^{\text {e }}$ & $16.43 \%$ & $8.15 \%$ & $49.46 \%$ & $22.13 \%$ & $2.09 \%$ & $1.49 \%$ & $3.43 \%$ & $1.36 \%$ \\
\hline run time $^{\mathrm{f}}$ & 0.24 & 0.37 & 0.30 & 0.40 & 0.32 & 0.62 & 0.48 & 0.42 \\
\hline
\end{tabular}

${ }^{\mathrm{a}}$ Minimum objective value obtained in 20 runs

${ }^{\mathrm{b}}$ Maximum objective value obtained in 20 runs

${ }^{c}$ Average objective value of 20 runs

${ }^{\mathrm{d}}$ Standard deviation of objective values of 20 runs

${ }^{\mathrm{e}}$ Average percentage deviation from the best known solution

${ }^{\mathrm{f}}$ Average CPU time in minutes for each run

The search using only the operator of randomly swapping reversed subsequences may be too diversified, and may not lead to promising regions of the solution space. Thus, instead of using only one operator we will use a combination of several operators. A number of different combinations of operators have been tried and experimented with. The combination of the following operators seems to yield the most promising results: random swaps (swap), reversing a subsequence (reverse) and random swaps of reversed subsequences (swap_reverse). This seems reasonable as two of the operators were identified to yield the best results from Table 1, while the search using the third operator (i.e., random swapping) is not as diversified as the searches using the two other operators. Equal probabilities are associated with the operators being selected. Using this combination of operators, the heuristic was run and obtained an average deviation of $1.36 \%$ from the best known solution, as shown in the last column (combined) of Table 1. In addition, it is also faster than the variant using randomly swapping of reversed subsequences (swap_reverse) as the neighborhood operator.

Figure 9 shows the plot of the best objective solution values found by the heuristics using the operator of reversing a subsequence (reverse), the operator of randomly swapping of reversed 
subsequences (swap_reverse) and the combination of the operators for randomly swapping two positions, reversing a subsequence, and randomly swapping reversed subsequences (combined) during one run on instance vrpnc1. It can be observed from the figure that the variant using the operator of reversing a subsequence converges faster than the other two. However, the solution quality is not good as reflected by the objective value, which is the highest among the three operators. The operator of randomly swapping reversed subsequences converges at a later stage but achieves a better solution. The combined approach leads to the best solution quality than the other two, and the convergence rate is somewhere between the other two. This phenomenon was also observed when the different variants of the heuristic were experimented on the rest of the test instances. For this reason, the combination of the three different operators will be adopted in the heuristic to generate neighbor solutions.

\subsection{Calibrating limit}

As mentioned earlier, a food source $x_{i}$ will be abandoned if no improving neighbor solutions $\hat{x}$ can be found in the neighborhood of $x_{i}$ for consecutive limit iterations. Karaboga (2009) has shown that this parameter is important regarding the performance of the $\mathrm{ABC}$ algorithm on solving function optimization problems. Hence, we will also study the effect of this parameter on the performance of the $\mathrm{ABC}$ algorithm for solving the CVRP.

The value of the parameter limit was determined by running every benchmark instance 20 times for each of the predetermined values of limit. This calibrating process is important because if too few iterations are spent on exploring the neighborhood of a food source, the neighborhood may not be fully explored and the search will be too diversified. On the contrary, if too many iterations are spent on exploring the neighborhood, then the search will tend to focus on a few portions of the search space and will miss out other potential regions of the search space. Experiments on all the test instances show that the most appropriate value for the parameter limit is proportional to the number of customers $(n)$, and this value is approximately equal to $50 n$. Due to space limitation, we only show the average objective function values of instance vrpnc1 with 50 customers for the different values of limit in Figure 10. From this figure, we can easily see that limit $=2,500$ yields the best results. Thus limit $=50 n$ was used in all the experiments reported in the remainder of this section. 


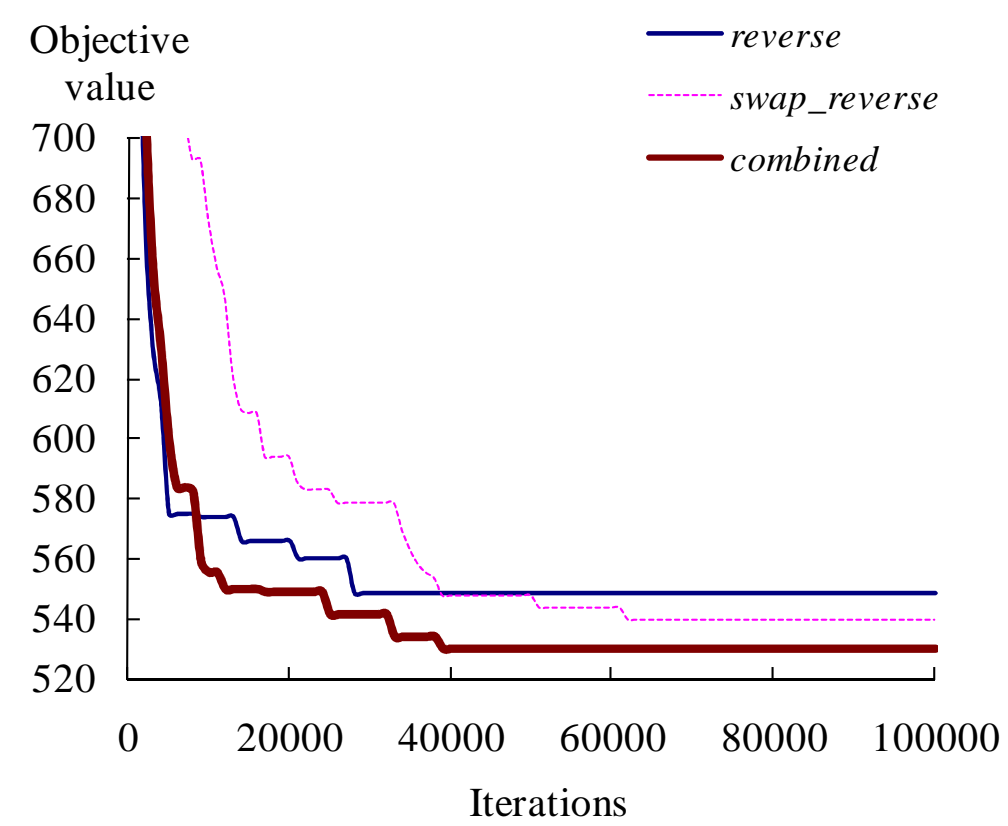

Figure 9. Converging processes for reverse, swap_reverse, and combined

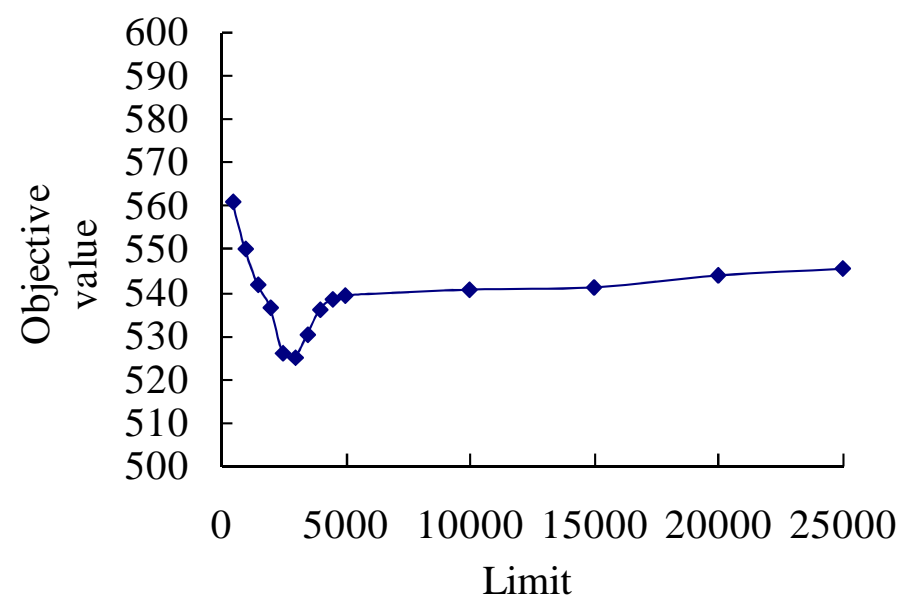

Figure 10. Effect of the parameter limit

\subsection{Original ABC vs. enhanced ABC}

According to some preliminary tests (not shown here) and the results shown in Table 1, the results of the CVRP obtained from the basic $\mathrm{ABC}$ algorithm are not very close to the best known result on average, even if we used many different combinations of operators. Therefore, we aim to improve the performance of the algorithm and propose an enhanced version. This version has been depicted in Section 3.6. The performance of this enhanced version was compared with that of the basic version. Both versions were implemented by using the combination of the three previously 
described operators as well as setting limit equal to 50n, and assessed by the set of 14 classical instances. Each test instance was run 20 times using each of the $\mathrm{ABC}$ heuristics. It was found that the speed of convergence of the two heuristics depends on $n$. Moreover, it was found that setting the termination condition (MaxIterations) to 2000 n iterations is sufficient for the two heuristics to converge. The best results, the average results, the average CPU times obtained by the two versions for each test instance are also reported in Table 2, where the solution values recorded for both the best and average results are rounded to the nearest integer. According to this table, the enhanced version obtained better solutions than the original version in all test instances in terms of both the average and the best results in 20 runs. The mean percentage improvement of the average (best) results of all test instances is $4.16 \%$ (3.53\%). The largest percentage improvement of the average (best) result is $12.29 \%(11.32 \%)$, indicating that the enhanced version can produce much better solutions than the basic version. From the table, we can observe that the enhanced ABC heuristic requires more computational effort than the original version. This is due to the modification of step $4 \mathrm{~d}$ of the enhanced heuristic.

Table 2. Comparison of experimental results between the original and enhanced ABC heuristics

\begin{tabular}{|c|c|c|c|c|c|c|c|}
\hline \multirow[t]{2}{*}{ Instance } & \multicolumn{3}{|c|}{ Original $\mathrm{ABC}$} & \multicolumn{3}{|c|}{ Enhanced ABC } & \multirow[t]{2}{*}{ Improvement $^{\mathrm{d}}$} \\
\hline & Best $^{\mathrm{a}}$ & $A v g^{\mathrm{b}}$ & $C P U^{\mathrm{c}}$ & Best $^{\mathrm{a}}$ & $A v g .^{b}$ & $C P U^{\mathrm{c}}$ & \\
\hline vrp_nc1 & 526.97 & 531.74 & 0.42 & 524.61 & 526.23 & 0.65 & $1.04 \%$ \\
\hline vrp_nc2 & 865.23 & 885.78 & 0.85 & 836.74 & 842.97 & 1.33 & $4.83 \%$ \\
\hline vrp_nc3 & 842.47 & 850.20 & 0.94 & 831.16 & 834.61 & 1.68 & $1.83 \%$ \\
\hline vrp_nc4 & 1065.71 & 1087.29 & 5.17 & 1031.69 & 1061.80 & 8.17 & $2.34 \%$ \\
\hline vrp_nc5 & 1382.85 & 1398.04 & 5.84 & 1320.24 & 1331.24 & 9.72 & $4.78 \%$ \\
\hline vrp_nc6 & 560.33 & 570.48 & 0.44 & 555.43 & 558.09 & 0.70 & $2.17 \%$ \\
\hline vrp_nc7 & 945.56 & 958.87 & 1.86 & 909.68 & 916.58 & 2.35 & $4.41 \%$ \\
\hline vrp_nc8 & 887.71 & 897.44 & 1.94 & 865.94 & 876.12 & 2.54 & $2.38 \%$ \\
\hline vrp_nc9 & 1235.37 & 1265.80 & 4.88 & 1170.25 & 1192.64 & 6.93 & $5.78 \%$ \\
\hline vrp_nc10 & 1497.92 & 1517.24 & 8.59 & 1415.23 & 1434.05 & 12.83 & $5.48 \%$ \\
\hline vrp_nc11 & 1183.92 & 1203.29 & 2.20 & 1049.91 & 1055.41 & 3.47 & $12.29 \%$ \\
\hline vrp_nc12 & 843.17 & 854.32 & 2.07 & 819.56 & 821.37 & 2.90 & $3.86 \%$ \\
\hline vrp_nc13 & 1592.88 & 1623.29 & 3.53 & 1545.98 & 1558.28 & 4.97 & $4.00 \%$ \\
\hline vrp_nc14 & 881.41 & 895.27 & 2.62 & 866.37 & 868.60 & 3.35 & $2.98 \%$ \\
\hline
\end{tabular}

${ }^{\mathrm{a}}$ Best result obtained in 20 runs

${ }^{\mathrm{b}}$ Average result obtained in 20 runs

${ }^{c}$ Average CPU run time in minutes in 20 runs

${ }^{\mathrm{d}}$ Percentage improvement of the average result obtained by the enhanced $\mathrm{ABC}$ over the original $\mathrm{ABC}$

To clearly illustrate the effect of the modifications in steps $4 \mathrm{~d}$ and $4 \mathrm{e}$ on the improvement of 
solution quality, we plot figures 11a and 11b. Figure 11a shows the plot of the best objective values found by the original $\mathrm{ABC}$ heuristic, the semi-enhanced $\mathrm{ABC}$ heuristic (where only step $4 \mathrm{e}$ is enhanced) and the enhanced $\mathrm{ABC}$ heuristic (both the steps $4 \mathrm{~d}$ and $4 \mathrm{e}$ are enhanced) during one run on instance vrpnc1. It can be observed from the figure that the original and semi-enhanced $\mathrm{ABC}$ heuristics converge at the same rate, while the semi-enhanced version obtains a better solution. The enhanced variant converges at an earlier stage with a better solution than the other two variants.

Figure 11b shows the plot of the average objective values of the $\tau$ food sources obtained by the original, semi-enhanced and enhanced $\mathrm{ABC}$ heuristics at the different iterations during one run. The behaviors of the semi-enhanced and enhanced $\mathrm{ABC}$ heuristics are similar where the values are gradually lowered. On the opposite, the average objective values of the original $\mathrm{ABC}$ heuristic do not show this pattern and some fluctuations can be observed. Around 10,000 iterations, the original $\mathrm{ABC}$ heuristic has identified a minimum average objective value. However, the algorithm has not been able to improve this further but has given some fluctuation results. This is due to step $4 \mathrm{e}$ where a food source $x_{i}$ will be replaced by a randomly generated solution if no improving neighbor solutions $\hat{x}$ have been encountered for consecutive limit iterations. The effect is that after most food sources have not been improved for limit iterations, the original ABC heuristic randomly generates new solutions in which most of the new solutions have large objective values compared with the minimum average objective value. The outcome is that the average objective value increases after reaching the minimum average. The average objective value decreases slightly afterwards because the heuristics finds most of the food sources near local minima. However, the average value increases again later due to creating many new food sources to avoid trapping in local minima. This process is repeated and this is why the fluctuation can be observed. On the contrary, the average objective values of the enhanced ABC heuristic are gradually lowered due to the fact that a food source is replaced by a neighbor solution and not by a randomly generated solution, where the neighbor solutions have higher chances to have closer objective values to the minimum average objective value especially if the local objective surface is not too mountainous. 


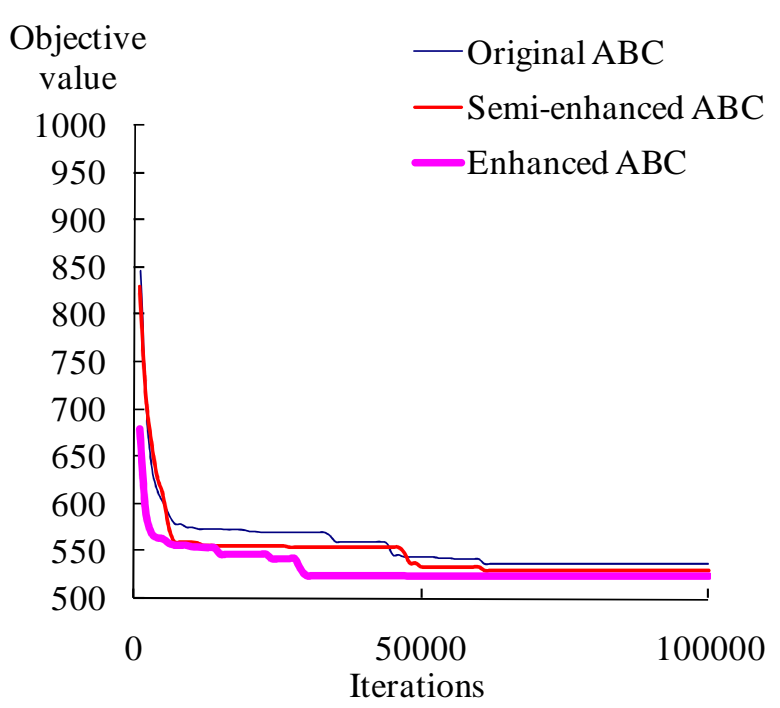

(a) Best solution

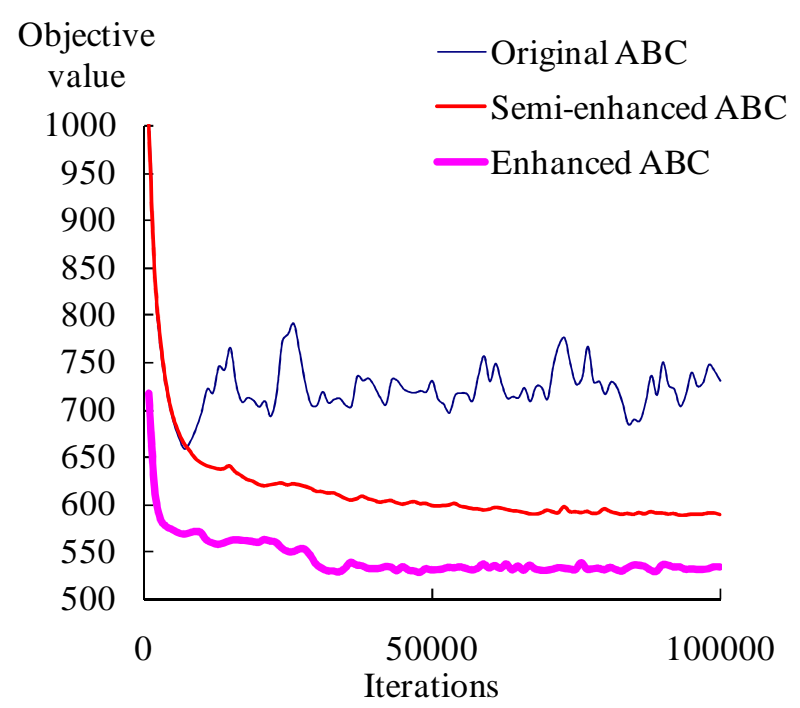

(b) Average solution

Figure 11. Converging processes of the basic $\mathrm{ABC}$ heuristic and its variants

In order to evaluate the effect of the modification made in step $4 \mathrm{~d}$ on the average performance, we compared the average objective values of the semi-enhanced $\mathrm{ABC}$ heuristic with those of the enhanced one in figure 11b. It can be observed that the average objective values of the semienhanced variant are much worse than those of the enhanced variant, which indicates that the modification made in step $4 \mathrm{~d}$ significantly improves the quality of the search.

\subsection{Computational results}

Table 3 lists the characteristics of the 14 classical instances. For each test instance, the table indicates the number of customers $(n)$, the vehicle capacity $(Q)$, the service time $(s)$ for each customer, the route maximum length $(L)$ and the number of vehicle routes $(m)$. The table also gives for each instance the best known solution so far. In addition, the table provides information about the average and best solutions obtained with the enhanced $A B C$ heuristic. It can be seen from the table that the enhanced $\mathrm{ABC}$ heuristic has matched six of the 14 best known solutions, and the average deviation from the best solutions is $0.46 \%$. 
Table 3. Enhanced $\mathrm{ABC}$ results for the classical instances

\begin{tabular}{lrlrrrrrrr}
\hline Instance & $n$ & $Q$ & \multicolumn{1}{l}{$l$} & $L$ & $m$ & $\begin{array}{r}\text { Best known } \\
\text { solution }\end{array}$ & $\begin{array}{r}\mathrm{ABC} \\
\text { average }^{\mathrm{d}}\end{array}$ & $\begin{array}{r}\mathrm{ABC} \\
\text { best }^{\mathrm{e}}\end{array}$ & $\begin{array}{r}\mathrm{DFB}^{\mathrm{f}} \\
(\%)\end{array}$ \\
\hline vrpnc1 & 50 & 160 & 0 & $\infty$ & 5 & $524.61^{\mathrm{a}}$ & 526.23 & $\mathbf{5 2 4 . 6 1}$ & 0.00 \\
vrpnc2 & 75 & 140 & 0 & $\infty$ & 10 & $835.26^{\mathrm{a}}$ & 842.97 & 836.74 & 0.18 \\
vrpnc3 & 100 & 200 & 0 & $\infty$ & 8 & $826.14^{\mathrm{a}}$ & 834.61 & 831.16 & 0.61 \\
vrpnc4 & 150 & 200 & 0 & $\infty$ & 12 & $1028.42^{\mathrm{a}}$ & 1061.80 & 1031.69 & 0.32 \\
vrpnc5 & 199 & 200 & 0 & $\infty$ & 17 & $1291.29^{\mathrm{b}}$ & 1331.24 & 1320.24 & 2.24 \\
vrpnc6 & 50 & 160 & 10 & 200 & 6 & $555.43^{\mathrm{a}}$ & 558.09 & $\mathbf{5 5 5 . 4 3}$ & 0.00 \\
vrpnc7 & 75 & 140 & 10 & 160 & 11 & $909.68^{\mathrm{a}}$ & 916.58 & $\mathbf{9 0 9 . 6 8}$ & 0.00 \\
vrpnc8 & 100 & 200 & 10 & 230 & 9 & $865.94^{\mathrm{a}}$ & 876.12 & $\mathbf{8 6 5 . 9 4}$ & 0.00 \\
vrpnc9 & 150 & 200 & 10 & 200 & 14 & $1162.55^{\mathrm{a}}$ & 1192.64 & 1170.25 & 0.66 \\
vrpnc10 & 199 & 200 & 10 & 200 & 18 & $1395.85^{\mathrm{c}}$ & 1434.05 & 1415.23 & 1.39 \\
vrpnc11 & 120 & 200 & 0 & $\infty$ & 7 & $1042.11^{\mathrm{a}}$ & 1055.41 & 1049.91 & 0.75 \\
vrpnc12 & 100 & 200 & 0 & $\infty$ & 10 & $819.56^{\mathrm{a}}$ & 821.37 & $\mathbf{8 1 9 . 5 6}$ & 0.00 \\
vrpnc13 & 120 & 200 & 50 & 720 & 11 & $1541.14^{\mathrm{a}}$ & 1558.28 & 1545.98 & 0.31 \\
vrpnc14 & 100 & 200 & 90 & 1040 & 11 & $866.37^{\mathrm{a}}$ & 868.60 & $\mathbf{8 6 6 . 3 7}$ & 0.00 \\
& & & & \multicolumn{7}{c}{ Average } & & & & 0.46 \\
\hline
\end{tabular}

${ }^{\text {a }}$ Obtained from Taillard (1993)

${ }^{\mathrm{b}}$ Obtained from Mester and Bräysy (2007)

${ }^{\mathrm{c}}$ Obtained from Rochat and Taillard (1995)

${ }^{\mathrm{d}}$ Average solution obtained by enhanced ABC in 20 runs

${ }^{\mathrm{e}}$ Best solution obtained by enhanced ABC in 20 runs

${ }^{\mathrm{f}}$ Deviation of $\mathrm{ABC}$ best from the best known solution

Table 4 provides a comparison on the results obtained on the 14 classical instances by some of the metaheuristics for the CVRP. It is clear that the enhanced ABC heuristic performs better than some of the well-known tabu search heuristics such as Taburoute (Gendreau et al., 1994), the ejection chain method (Rego and Roucairol, 1996), the subpath ejection chain method (Rego, 1998), the granular tabu search (Toth and Vigo, 2003) and the Unified Tabu Search (Cordeau et al., 2001). In addition, the enhanced $\mathrm{ABC}$ heuristic outperforms the simulated annealing heuristic by Osman (1993), the saving-based ant system of Bullnheimer et al. (1999) and Reimann et al. (2004), and the hybrid genetic algorithm of Berger and Barkaoui (2003). Finally, the enhanced ABC heuristic also surpasses the path relinking approach of Ho and Gendreau (2006), the particle swarm optimization method by Ai and Kachitvichyanukul (2009) and the hybrid eletromagnetism-like algorithm of Yurtkuran and Emel (2010). Outstanding results were obtained by the adaptive memory procedure of Rochat and Taillard (1995) and by the memetic heuristic by Nagata and Bräysy (2009). The performances of the honey bees mating optimization algorithm (Marinakis et al., 2010) and the 
AGES algorithm (Mester and Bräysy, 2007) are also excellent.

Table 4. Comparison of computational results of different methods for the classical instances

\begin{tabular}{|c|c|c|c|}
\hline Reference & $\mathrm{ADFB}^{\mathrm{a}}$ & CPU time $^{\mathrm{b}}$ & Computer \\
\hline Rochat and Taillard (1995) & 0.00 & N/A & Silicon Graphics $100 \mathrm{MHz}$ \\
\hline Nagata and Bräysy (2009) & 0.00 & 13.80 & Opteron $2.4 \mathrm{GHz}$ \\
\hline Mester and Bräysy (2007) & 0.03 & 2.80 & Pentium IV $2.8 \mathrm{GHz}$ \\
\hline Marinakis et al. (2010) & 0.03 & 0.80 & Pentium M750 1.86 GHz \\
\hline Taillard (1993) & 0.05 & N/A & Silicon Graphics $100 \mathrm{MHz}$ \\
\hline Prins (2009) & 0.07 & 0.27 & Pentium IV $2.8 \mathrm{GHz}$ \\
\hline Pisenger and Ropke (2007) & 0.11 & 17.50 & Pentium IV $3 \mathrm{GHz}$ \\
\hline Chen et al. (2010) & 0.13 & 10.90 & Pentium IV $2.93 \mathrm{GHz}$ \\
\hline Yu et al. (2009) & 0.14 & 2.91 & Pentium $1000 \mathrm{MHz}$ \\
\hline Tarantilis (2005) & 0.20 & 5.63 & Pentium 400 MHz \\
\hline Derigs and Kaiser (2007) & 0.21 & 5.84 & Celeron $2.4 \mathrm{GHz}$ \\
\hline Tarantilis and Kiranoudis (2002) & 0.23 & 5.22 & Pentium $2400 \mathrm{MHz}$ \\
\hline Ergun et al. (2006) & 0.23 & 28.91 & Pentium 733 MHz \\
\hline Prins (2004) & 0.24 & 5.19 & Pentium $1 \mathrm{GHz}$ \\
\hline Lin et al. (2009) & 0.35 & 8.21 & Pentium IV $2.8 \mathrm{GHz}$ \\
\hline Szeto et al. & 0.46 & 4.40 & Pentium $1.73 \mathrm{GHz}$ \\
\hline Reimann et al. (2004) & 0.48 & 3.63 & Pentium $900 \mathrm{MHz}$ \\
\hline Berger and Barkaoui (2003) & 0.49 & 21.25 & Pentium $400 \mathrm{MHz}$ \\
\hline Ho and Gendreau (2006) & 0.54 & 4.13 & Pentium $2.53 \mathrm{GHz}$ \\
\hline Rego and Roucairol (1996) & 0.55 & 24.65 & 4 Sun Sparc IPC \\
\hline Cordeau et al. (2001) & $0.56^{\mathrm{c}}$ & 24.62 & Pentium IV 2 GHz \\
\hline Baker and Ayechew (2003) & 0.56 & 29.11 & Pentium $266 \mathrm{MHz}$ \\
\hline Toth and Vigo (2003) & 0.64 & 3.84 & Pentium $200 \mathrm{MHz}$ \\
\hline Gendreau et al. (1994) & 0.86 & 46.80 & Silicon Graphics $36 \mathrm{MHz}$ \\
\hline Ai and Kachitvichyanukul (2009) & 0.88 & 2.72 & Intel P4 $3.4 \mathrm{GHz}$ \\
\hline Yurtkuran and Emel (2010) & 1.04 & 2.20 & Intel Core2 Duo, $2 \mathrm{GHz}$ \\
\hline Bullnheimer et al. (1999) & 1.51 & 18.44 & Pentium $100 \mathrm{MHz}$ \\
\hline Rego (1998) & 1.54 & 2.32 & HP 9000/712 \\
\hline Osman (1993) & 2.11 & 151.35 & VAX 8600 \\
\hline
\end{tabular}

${ }^{\text {a }}$ Average deviation from best known results

${ }^{\mathrm{b}}$ Average computing time in minutes

${ }^{\mathrm{c}}$ Computational results obtained from Cordeau et al. (2005)

Many of the heuristics within the $0.1 \%$ average deviation from best known solutions are populationbased methods combined with local search, and many of the methods that yielded an average deviation of less than $0.4 \%$ are hybrids of various techniques. The implemented version of the heuristic is a pure artificial bee colony algorithm, and is also one of the effective algorithms for the CVRP. It is not easy to compare running times for the various metaheuristics due to different 
computing environments. We believe that the reported CPU time for the enhanced ABC heuristic is reasonable and acceptable.

Table 5. Enhanced ABC results for the large-scale instances

\begin{tabular}{lcrrrrrrr}
\hline Instance & $n$ & $Q$ & $s$ & $L$ & $m$ & $\begin{array}{r}\text { Best known } \\
\text { solution }\end{array}$ & $\begin{array}{r}\text { ABC } \\
\text { solution }\end{array}$ & $\begin{array}{r}\text { DFB }^{\mathrm{d}} \\
(\%)\end{array}$ \\
\hline kelly01 & 240 & 550 & 0 & 650 & 10 & $5626.81^{\mathrm{a}}$ & 5861.14 & 4.16 \\
kelly02 & 320 & 700 & 0 & 900 & 11 & $8431.66^{\mathrm{a}}$ & 8720.37 & 3.42 \\
kelly03 & 400 & 900 & 0 & 1200 & 10 & $11036.22^{\mathrm{b}}$ & 11572.69 & 4.86 \\
kelly04 & 480 & 1000 & 0 & 1600 & 10 & $13592.88^{\mathrm{a}}$ & 13829.02 & 1.74 \\
kelly05 & 200 & 900 & 0 & 1800 & 5 & $6460.98^{\mathrm{b}}$ & 6593.17 & 2.05 \\
kelly06 & 280 & 900 & 0 & 1500 & 7 & $8404.26^{\mathrm{a}}$ & 8729.70 & 3.87 \\
kelly07 & 360 & 900 & 0 & 1300 & 9 & $10156.58^{\mathrm{a}}$ & 10533.43 & 3.71 \\
kelly08 & 440 & 900 & 0 & 1200 & 11 & $11643.90^{\mathrm{c}}$ & 12054.77 & 3.53 \\
kelly09 & 255 & 1000 & 0 & $\infty$ & 14 & $580.42^{\mathrm{a}}$ & 593.35 & 2.23 \\
kelly10 & 323 & 1000 & 0 & $\infty$ & 16 & $738.49^{\mathrm{a}}$ & 750.77 & 1.66 \\
kelly11 & 399 & 1000 & 0 & $\infty$ & 18 & $914.72^{\mathrm{a}}$ & 932.81 & 1.98 \\
kelly12 & 483 & 1000 & 0 & $\infty$ & 19 & $1106.76^{\mathrm{a}}$ & 1138.32 & 2.85 \\
kelly13 & 252 & 1000 & 0 & $\infty$ & 26 & $857.19^{\mathrm{a}}$ & 872.45 & 1.78 \\
kelly14 & 320 & 1000 & 0 & $\infty$ & 30 & $1080.55^{\mathrm{a}}$ & 1109.48 & 2.68 \\
kelly15 & 396 & 1000 & 0 & $\infty$ & 33 & $1342.53^{\mathrm{a}}$ & 1367.37 & 1.85 \\
kelly16 & 480 & 1000 & 0 & $\infty$ & 37 & $1620.85^{\mathrm{a}}$ & 1651.31 & 1.88 \\
kelly17 & 240 & 200 & 0 & $\infty$ & 22 & $707.76^{\mathrm{a}}$ & 710.42 & 0.36 \\
kelly18 & 300 & 200 & 0 & $\infty$ & 27 & $995.13^{\mathrm{a}}$ & 1019.64 & 2.46 \\
kelly19 & 360 & 200 & 0 & $\infty$ & 33 & $1365.97^{\mathrm{a}}$ & 1377.54 & 0.85 \\
kelly20 & 420 & 200 & 0 & $\infty$ & 38 & $1820.02^{\mathrm{a}}$ & 1850.59 & 1.68 \\
\hline & & & & & Average & & 2.48 \\
\hline
\end{tabular}

${ }^{a}$ Obtained from Nagata and Bräysy (2009)

${ }^{\mathrm{b}}$ Obtained from Prins (2004)

${ }^{\mathrm{c}}$ Obtained from Prins (2009)

${ }^{\mathrm{d}}$ Deviation from the best known solution

The enhanced ABC heuristic was also tested on the 20 large-scale instances by Golden et al. (1998). Table 5 lists the characteristics of these instances together with the average solutions and best solutions obtained by the enhanced $\mathrm{ABC}$ heuristic. The average deviation from best known solutions is $2.31 \%$. Table 6 provides a comparison on the results with some of the metaheuristics published in the literature. The enhanced ABC heuristic outperforms the deterministic annealing algorithm described in Golden et al. (1998), the granular tabu search by Toth and Vigo (2003), the very large neighborhood search by Ergun et al. (2006), the path relinking approach by Ho and Gendreau (2006) and the variable neighborhood search procedure by Kytöjoki et al. (2007). As with 
the classical instances, population-based heuristics combined with local search also generated the best results for the large-scale instances. From the table we notice that the memetic algorithm by Nagata and Bräysy (2009) and the AGES algorithm by Mester and Bräysy (2007) obtained much better solutions than the rest of the methods.

Table 6. Comparison of computational results of different methods for the large-

\begin{tabular}{|c|c|c|c|}
\hline \multicolumn{4}{|c|}{ scale instances } \\
\hline Reference & $\mathrm{ADFB}^{\mathrm{a}}$ & CPU time ${ }^{b}$ & Computer \\
\hline Nagata and Bräysy (2009) & 0.02 & 355.90 & Opteron $2.4 \mathrm{GHz}$ \\
\hline Mester and Bräysy (2007) & 0.18 & 24.40 & Pentium IV $2.8 \mathrm{GHz}$ \\
\hline Prins (2009) & 0.48 & 7.27 & Pentium IV $2.8 \mathrm{GHz}$ \\
\hline Marinakis et al. (2010) & 0.58 & 4.06 & Pentium M750 1.86 GHz \\
\hline Pisinger and Ropke (2007) & 0.67 & 107.67 & Pentium IV 3 GHz \\
\hline Chen et al. (2010) & 0.74 & 284.40 & Pentium IV $2.93 \mathrm{GHz}$ \\
\hline Reimann et al. (2004) & 0.78 & 49.33 & Pentium $900 \mathrm{MHz}$ \\
\hline Tarantilis (2005) & 0.78 & 45.58 & Pentium $400 \mathrm{MHz}$ \\
\hline Derigs and Kaiser (2007) & 0.86 & 113.34 & Celeron $2.4 \mathrm{GHz}$ \\
\hline Prins $(2004)$ & 1.09 & 66.90 & Pentium $1 \mathrm{GHz}$ \\
\hline Li et al. (2005) & 1.24 & N/A & N/A \\
\hline Cordeau et al. (2001) & $1.64^{\mathrm{c}}$ & 56.11 & Pentium $2 \mathrm{GHz}$ \\
\hline Lin et al. (2009) & 1.79 & 118.98 & Pentium IV $2.8 \mathrm{GHz}$ \\
\hline Szeto et al. & 2.48 & 32.38 & Pentium $1.73 \mathrm{GHz}$ \\
\hline Ho and Gendreau (2006) & 2.74 & 39.96 & Pentium $2.53 \mathrm{GHz}$ \\
\hline Toth and Vigo (2003) & 3.06 & 17.55 & Pentium $200 \mathrm{MHz}$ \\
\hline Ergun et al. (2006) & 3.95 & 137.95 & Pentium $733 \mathrm{MHz}$ \\
\hline Golden et al. (1998) & 4.10 & 37.20 & Pentium $100 \mathrm{MHz}$ \\
\hline Kytöjoki et al. (2007) & 4.67 & 0.02 & AMD Athlon64 3000+ \\
\hline
\end{tabular}

${ }^{a}$ Average deviation from best known results

${ }^{\mathrm{b}}$ Average computing time in minutes

${ }^{\mathrm{c}}$ Computational results obtained from Cordeau et al. (2005)

\section{Conclusions}

In this paper, we presented an artificial bee colony (ABC) heuristic for the capacitated vehicle routing problem (CVRP). The ABC heuristic is a fairly new approach, and it is based on the foraging behavior of honey bees. An enhanced version of the $\mathrm{ABC}$ heuristic has been developed to improve the original heuristic for solving the CVRP. Computational results show that the enhanced $\mathrm{ABC}$ heuristic is able to produce much better solutions than the original one and uses slightly little more computation time. The results also show that the enhanced $\mathrm{ABC}$ can produce good solutions when compared with existing heuristics for solving the CVRP. These results seem to indicate that the enhanced heuristic is an alternative to solve the capacitated vehicle routing problem. 


\section{Acknowledgements}

The research was jointly supported by a grant (200902172003) from the Hui Oi Chow Trust Fund and two grants (201001159008 and 201011159026) from the University Research Committee of the University of Hong Kong. This support is gratefully acknowledged. Thanks are also due to the three anonymous referees for their valuable comments.

\section{References}

Ai TJ, Kachitvichyanukul V. Particle swarm optimization and two solution representations for solving the capacitated vehicle routing problem. Computers and Industrial Engineering 2009; 56; $380-387$.

Baker BM, Ayechew MA. A genetic algorithm for the vehicle routing problem. Computers \& Operations Research 2003; 30; 787-800.

Baldacci R, Toth P, Vigo D. Exact algorithms for routing problems under vehicle capacity constraints. Annals of Operations Research 2010; 175; 213-245.

Baykasoğlu A, Özbakir L, Tapkan P 2007. Artificial bee colony algorithm and its application to generalized assignment problem. In: Chan FTS, Tiwari MK (Eds.). Swarm Intelligence: Focus on Ant and Particle Swarm Optimization. Vienna, Itech Education and Publishing; 2007. p. 113144.

Berger J, Barkoui M. A new hybrid genetic algorithm for the capacitated vehicle routing problem. Journal of the Operational Research Society 2003; 54; 1254-1262.

Bullnheimer B, Hartl RF, Strauss C. An improved ant system algorithm for the vehicle routing problem. Annals of Operations Research 1999; 89; 319-328.

Chen $\mathrm{P}$, Huang $\mathrm{H}-\mathrm{k}$, Dong $\mathrm{X}-\mathrm{Y}$. Iterated variable neighborhood descent algorithm for the capacitated vehicle routing problem. Expert Systems with Applications 2010; 37; 1620-1627.

Christofides N, Eilon S. An algorithm for the vehicle dispatching problem. Operational Research Quartely 1969; 20; 309-318.

Christofides N, Mingozzi A, Toth P 1979. The vehicle routing problem. In: Christofides N, Mingozzi A, Toth P, Sandi C (Eds.). Combinatorial Optimization. Chichester, Wiley; 1979. p. 315-338.

Cordeau J-F, Gendreau M, Hertz A, Laporte G, Sormany J-S 2005. New heuristics for the vehicle routing problem. In: Langevin A, Riopel D (Eds.). Logistics Systems: Design and Optimization. Kluwer; 2005. p. 279-297. 
Cordeau J-F, Laporte G, Mercier A. A unified tabu search heuristic for vehicle routing problems with time windows. Journal of the Operational Research Society 2001; 52; 928-936.

Derigs U, Kaiser R. Applying the attribute based hill climber heuristic to the vehicle routing problem. European Journal of Operational Research 2007; 177; 719-732.

Ergun Ö, Orlin JB, Steele-Feldman A. Creating very large scale neighborhoods out of smaller ones by compounding moves. Journal of Heuristics 2006; 12; 115-140.

Gendreau M, Hertz A, Laporte G. A tabu search heuristic for the vehicle routing problem. Management Science 1994; 40; 1276-1290

Gendreau M, Laporte G, Potvin J-Y 2002. Metaheuristics for the capacitated VRP. In: Toth P, Vigo D (Eds.). The Vehicle Routing Problem. SIAM Society for Industrial and Applied Mathematics; 2002. p. 129-154.

Gendreau M, Potvin J-Y, Bräysy O, Hasle G, Løkketangen A 2008. Metaheuristics for the vehicle routing and its extensions: A categorized bibliography. In: Golden B, Raghavan S, Wasil E (Eds.). The vehicle routing problem: Latest advances and new challenges. Springer; 2008. p. 143-169.

Golden BL, Wasil EA, Kelly JP, Chao I-M 1998. Metaheuristics in vehicle routing. In: Crainic TG, Laporte G (Eds.). Fleet Management and Logistics. Kluwer; 1998. p. 33-56.

Ho SC, Gendreau M. Path relinking for the vehicle routing problem. Journal of Heuristics 2006; 12; $55-72$.

Juan AA, Faulin J, Ruiz R, Barrios B, Caballé S. The SR-GCWS hybrid algorithm for solving the vehicle routing problem. Applied Soft Computing 2010; 10; 215-224.

Kang F, Li J, Xu Q. Structural inverse analysis by hybrid simplex artificial bee colony algorithms. Computers and Structures 2009; 87; 861-870.

Karaboga D. An idea based on honey bee swarm for numerical optimization. Technical Report TR06, Erciyes University; 2005.

Karaboga D, Basturk B. A powerful and efficient algorithm for numerical function optimization: artificial bee colony (ABC) algorithm. Journal of Global Optimization 2007; 39; 459-471.

Karaboga D, Basturk B. On the performance of artificial bee colony (ABC) algorithm. Applied Soft Computing 2008; 8; 687-697.

Karaboga D, Ozturk C. Neural networks training by artificial bee colony algorithm on pattern classification. Neural Network World 2009; 19; 279-292.

Karaboga N. A new design method based on artificial bee colony algorithm for digital IIR filters. Journal of the Franklin Institute 2009; 346; 328-348.

Kytöjoki J, Nuortio T, Bräysy O, Gendreau M. An efficient variable neighborhood search heuristic 
for very large scale vehicle routing problems. Computers \& Operations Research 2007; 34; 2743-2757.

Li F, Golden B, Wasil E. Very large-scale vehicle routing: New test problems, algorithms, and results. Computers \& Operations Research 2005; 32; 1165-1179.

Lin S-W, Lee Z-J, Ying K-C, Lee C-Y. Applying hybrid meta-heuristics for capacitated vehicle routing problem. Expert Systems with Applications 2009; 36; 1505-1512.

Marinakis Y, Marinaki M, Dounias G. Honey Bees Mating Optimization algorithm for large scale vehicle routing problems. Natural Computing 2010; 9; 5-27.

Mester D, Bräysy O. Active-guided evolution strategies for large-scale capacitated vehicle routing problems. Computers \& Operations Research 2007; 34; 2964-2975.

Nagata Y, Bräysy O. Edge assembly-based memetic algorithm for the capacitated vehicle routing problem. Networks 2009; 54; 205-215.

Osman IH. Metastrategy simulated annealing and tabu search algorithms for the vehicle routing problem. Annals of Operations Research 1993; 41; 421-451.

Pisinger D, Ropke S. A general heuristic for vehicle routing problems. Computers \& Operations Research 2007; 34; 2403-2435.

Prins C. A simple and effective evolutionary algorithm for the vehicle routing problem. Computers \& Operations Research 2004; 31; 1985-2002.

Prins C 2009. A GRASP x Evolutionary local search hybrid for the vehicle routing problem. In: Pereira FB, Tavares J (Eds.). Bio-inspired Algorithms for the Vehicle Routing Problem. Springer-Verlag Berlin Heidelberg; 2009. p. 35-53.

Reimann M, Doerner K, Hartl RF. D-Ants: Savings Based Ants divide and conquer the vehicle routing problem. Computers \& Operations Research 2004; 31; 563-591.

Rego C. A subpath ejection method for the vehicle routing problem. Management Science 1998; 44; 1447-1459.

Rego C, Roucairol C 1996. A parallel tabu search algorithm using ejection chains for the vehicle routing problem. In: Osman IH, Kelly P (Eds.). Meta-Heuristics: Theory and Applications, Boston, Kluwer; 1996. p. 661-675.

Rochat Y, Taillard É. Probabilistic diversification and intensification in local search for vehicle routing. Journal of Heuristics 1995; 1; 147-167.

Singh A. An artificial bee colony algorithm for the leaf-constrained minimum spanning tree problem. Applied Soft Computing 2009; 9; 625-631.

Taillard É. Parallel iterative search methods for vehicle routing problems. Networks 1993; 23; 661673. 
Tarantilis CD. Solving the vehicle routing problem with adaptive memory programming methodology. Computers \& Operations Research 2005; 32; 2309-2327.

Tarantilis CD, Kiranoudis CT. BoneRoute: An adaptive memory-based method for effective fleet management. Annals of Operations Research 2002; 115; 227-241.

Toth P, Vigo D (Eds.). The Vehicle Routing Problem. SIAM Society for Industrial and Applied Mathematics, Philadelphia; 2002.

Toth P, Vigo D. The granular tabu search and its application to the vehicle-routing problem. Journal on Computing 2003; 15; 333-346.

Yu B, Yang Z-Z, Yao B. An improved ant colony optimization for vehicle routing problem. European Journal of Operational Research 2009; 196; 171-176.

Yurtkuran A, Emel E. A new hybrid electromagnetism-like algorithm for capacitated vehicle routing problems. Expert Systems with Applications 2010; 37; 3427-3433. 